\title{
Intermolecular Singlet Fission in Unsymmetrical Derivatives of Pentacene in Solution
}

Rubén Casillas, Matthias Adam, Pedro B. Coto*, Andreas R. Waterloo, Johannes Zirzlmeier, S. Rajagopala Reddy, Frank Hampel, Robert McDonald, Rik R. Tykwinski*, Michael Thoss*, and Dirk M. Guldi*

M. Sc. Rubén Casillas, Dr. Johannes Zirzlmeier, Prof. Dirk. M. Guldi

Friedrich-Alexander-Universität Erlangen-Nürnberg, Department of Chemistry and Pharmacy

\& Center of Excellence Engineering of Advanced Materials, Egerlandstrasse 3, 91058,

Erlangen, Germany

E-mail: dirk.guldi@fau.de

Matthias Adam, Dr. Andreas R. Waterloo, Dr. Frank Hampel

Department of Chemistry and Pharmacy \& Interdisciplinary Center for Molecular Materials

(ICMM), Friedrich-Alexander-Universität (FAU), Nikolaus-Fiebiger-Strasse 10, 91058

Erlangen, Germany

Dr. Pedro B. Coto

Friedrich-Alexander-Universität Erlangen-Nürnberg, Institute for Theoretical Physics,

Staudtstrasse 7 / B2, 91058 Erlangen, Germany

University of Oviedo, Department of Physical and Analytical Chemistry

Julián Clavería 8, 33006 Oviedo, Spain

E-mail: branapedro@uniovi.es

Dr. S. Rajagopala Reddy

Unité de Chimie Physique Théorique et Structurale, Namur Institute of Structured Matter,

University of Namur, 61 Rue de Bruxelles, B-5000 Namur, Belgium

Dr. Robert McDonald, Prof. Rik R. Tykwinski

Department of Chemistry, University of Alberta, Edmonton, Alberta T6G 2G2, Canada

E-mail: rik.tykwinski@ualberta.ca

Prof. Michael Thoss

Albert-Ludwigs-Universität Freiburg, Institute of Physics, Hermann-Herder-Strasse 3, 79104

Freiburg, Germany

E-mail: michael.thoss@ physik.uni-erlangen.de

Keywords: singlet fission, triplet diffusion, excitonic intermediate, unsymmetrical pentacene

Abstract: Intermolecular singlet fission (SF) is an electronically coupled process between two chromophores, where distance dependences are decisive in terms of rates and yields. In the current work, a family of pentacene derivatives featuring different functional groups have been designed, synthesized, and probed with respect to intermolecular SF in the low, medium, and high concentration regimes rather than in the solid state. By means of advanced photophysical techniques, global analysis modeling, and ab-initio calculations, we postulate a model for intermolecular SF. Our model is based on an early key intermediate, which involves the 
diffusional encounter between one pentacene in its singlet excited-state with another one in its ground state and which features excimer characteristics. This is followed by a transformation into a coupled triplet excited-state. The role of the functional group appended to pentacene was analyzed with respect to steric shielding of the pentacene core as a means to prevent photophysical degradation, as well as control diffusional encounter and, subsequently, SF. Our findings demonstrate the potential of new molecular materials for SF, especially in solution studies, as well as the challenges of implementing them in energy conversion schemes due the appearance of photodegradation processes that competes with SF.

\section{Introduction}

In the context of boosting the efficiency of conventional solar cells beyond the ShockleyQueisser limit, molecular materials that undergo singlet fission (SF) are attracting considerable attention. ${ }^{[1-4]}$ In SF, the absorption of one photon initiates the formation of a singlet excitedstate, which subsequently splits between neighboring chromophores into a pair of lower energy triplet excited-states. ${ }^{[5-8]}$

A number of requirements to activate SF are well understood, while some mechanistic aspects are controversially debate. ${ }^{[9-11]}$ Recent works have, for example, identified energy relationships that electronic excited-states must fulfill, namely (i) $\mathrm{E}\left(\mathrm{S}_{1}\right) \geq 2 \mathrm{E}\left(\mathrm{T}_{1}\right)$ and (ii) $\mathrm{E}\left(\mathrm{T}_{2}\right) \geq 2 \mathrm{E}\left(\mathrm{T}_{1}\right)$, for SF to be exoergic and to minimize quenching, via triplet-triplet annihilation. ${ }^{[6,12,13]}$ To date, SF has been observed in polycrystalline and amorphous films, polymers, as well as in dilute and concentrated solutions. ${ }^{[14-23]}$ It is now widely accepted that SF is possible as long as the electronic coupling between two or more chromophores is sufficiently strong, but, not be too strong. ${ }^{[24,25]}$ A favorable configuration between two chromophores is crucial to achieve the appropriate coupling for intermolecular SF and between two chromophore units for intramolecular SF. ${ }^{[6,9]}$ In particular, $\pi-\pi$ interactions facilitate the geometrical arrangement 
and/or stacking of the chromophores that, in turn, maximize the electronic coupling in the excited-states. ${ }^{[20,26-28]}$

In the current work, we focus on a group of pentacene derivatives with substituents that vary in size and constitution, namely (TIPS)2-Pn, TIPS-Pn-Me, and TIPS-Pn-H (Figure 1), to investigate the effects of functionalization and concentration on intermolecular SF in solution. We emphasize that the present study is focused on SF in solution, not in the solid state. In particular, novel synthetic approaches, photophysical assays, global analysis modeling, and $a b$ initio quantum chemistry calculations have been exploited to provide a convincing picture on how pentacene functionalization and the nature of the substituents influence the pathway of SF. Notably, assays in the concentration range of $1.0 \times 10^{-4}$ to $1.0 \times 10^{-1} \mathrm{M}$ shed light on diffusive and non-diffusive steps in the solution-based intermolecular SF, namely the formation and interaction of correlated triplet excited-states via diffusional encounter, dissociation into free, uncorrelated triplet excited-states, as well as deactivation processes such as photo-induced cyclization.

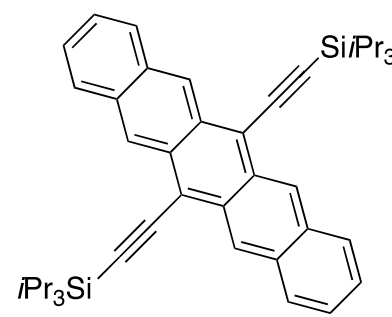

(TIPS)2-Pn

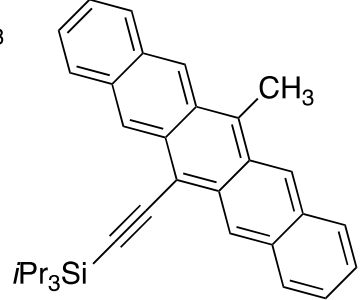

TIPS-Pn-Me

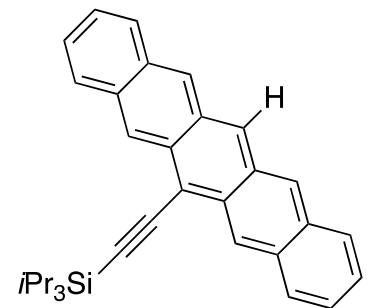

TIPS-Pn-H

Figure 1. Structures of (TIPS)2-Pn, TIPS-Pn-Me, and TIPS-Pn-H.

\section{Synthesis of Pentacene Derivatives}

The synthesis for (TIPS)2-Pn, TIPS-Pn-Me, and TIPS-Pn-H, (Figure 1) as well as the X-ray crystallographic analysis for TIPS-Pn-Me and TIPS-Pn-H have been provided in the supporting information (SI). In short, the synthesis of TIPS-Pn-Me was achieved via the established synthetic protocols developed to produce pentacene derivatives with unsymmetrical 
substitution in the 6- and 13-positions. ${ }^{[29-31]}$ The synthesis of TIPS-Pn-H was accomplished by reduction of ketone 1 using an excess of $\mathrm{LiAlH}_{4}$, followed by reductive aromatization using $\mathrm{SnCl}_{2}$ under acidic conditions (Figure 2) ${ }^{[32]}$ TIPS-Pn-H showed significantly lower stability in solution towards possible side-reactions compared to (TIPS)2-Pn and TIPS-Pn-Me, with dimer 2 being the major side product (see SI for details). Dimer 2 resulted from a cycloaddition reaction, which could be minimized by shielding solutions of 1 from ambient light during synthesis. TIPS-Pn-Me degraded slowly in solution in the presence of light and air to give the 6,13-endoperoxide (see SI for details), which could be prevented by storing the sample in deoxygenated solutions. In contrast, both TIPS-Pn-Me and TIPS-Pn-H were stable as solids for months when stored under refrigeration.
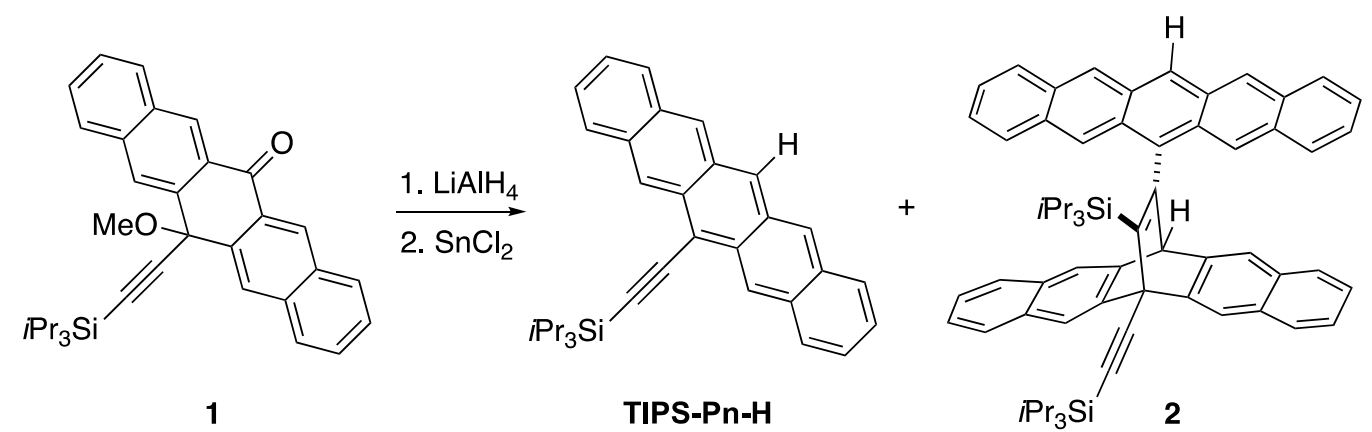

Figure 2. Reaction scheme for the synthesis of TIPS-Pn-H and structure of dimer 2.

\section{Photophysical Characterization in the Low Concentration Regime of $1.0 \times 10^{-4} \mathrm{M}$}

Initially, steady-state spectroscopy was used to examine the energetics related to the first singlet excited-states of (TIPS)2-Pn, TIPS-Pn-H, and TIPS-Pn-Me. In particular, the absorption and fluorescence spectra in the low concentration regime of $1.0 \times 10^{-4} \mathrm{M}$ are dominated by vibrational fine structure stemming from $0-0 *$ and $0-1 *$ as well as $* 1-0$ and $* 0-0$ transitions (Figure S11). From the fundamental $0-0^{*}$ absorption and fluorescence transitions, singlet excited-state energies of 1.94, 2.03, and $2.03 \mathrm{eV}$ were determined for (TIPS)2-Pn, TIPS-PnH, and TIPS-Pn-Me, respectively. Only (TIPS)2-Pn revealed vibrationally well-resolved 
absorption spectra. This is in stark contrast to TIPS-Pn-H and TIPS-Pn-Me, for which spectral broadening goes hand-in-hand with the loss of vibrational fine structure (Figure S11). ${ }^{[25]}$

We examined the triplet excited-state energy by means of low-temperature phosphorescence measurements. In this respect, we recently reported that the phosphorescence spectra of (TIPS)2-Pn showed a maximum at $1610 \mathrm{~nm}$, which relates to a triplet excited-state energy of $0.77 \mathrm{eV} .{ }^{[20]}$ Similarly, we identified a triplet excited-state energy for TIPS-Pn-Me of $0.76 \mathrm{eV}$, and a similar value has been calculated for the triplet excited-state of TIPS-Pn-H via ab-initio calculations - vide infra. From the corresponding singlet and triplet excited-state energies, the exergonic driving force for SF is $-0.41 \mathrm{eV}$ for (TIPS) 2 -Pn, $-0.51 \mathrm{eV}$ for TIPS-Pn-Me, and $-0.51 \mathrm{eV}$ for TIPS-Pn-H.

To evaluate the singlet excited-state decay and the corresponding triplet excited-state growth, we turned to nanosecond transient absorption studies (nsTA). In the nsTA analysis of TIPSPn-Me and (TIPS)2-Pn, the analogous fingerprint absorptions of their singlet excited-states are discernable immediately following excitation at $530 \mathrm{~nm} \cdot{ }^{[26]}$ Importantly, the minima match the vibrational fine structure seen in the ground-state absorption and, in turn, we attribute these minima to ground state bleach. From experiments at low concentrations $\left(1.0 \times 10^{-4} \mathrm{M}\right)$, singlet excited-state lifetimes of $13.3 \pm 0.4,12.0 \pm 0.5$, and $10.3 \pm 0.5 \mathrm{~ns}$ were derived from multiwavelength analyses for TIPS-Pn-H, TIPS-Pn-Me, and (TIPS)2-Pn, respectively (Figure S14). Following these decays, only the corresponding triplet excited-state features were present. For TIPS-Pn-H and TIPS-Pn-Me, the characteristic triplet excited-state fingerprints are maxima at 445 and $497 \mathrm{~nm}$, as well as minima at 580 and $642 \mathrm{~nm}$. Similarly, (TIPS)2-Pn gives rise to maxima at 466 and $500 \mathrm{~nm}$, followed by minima at 590 and $642 \mathrm{~nm}$. 

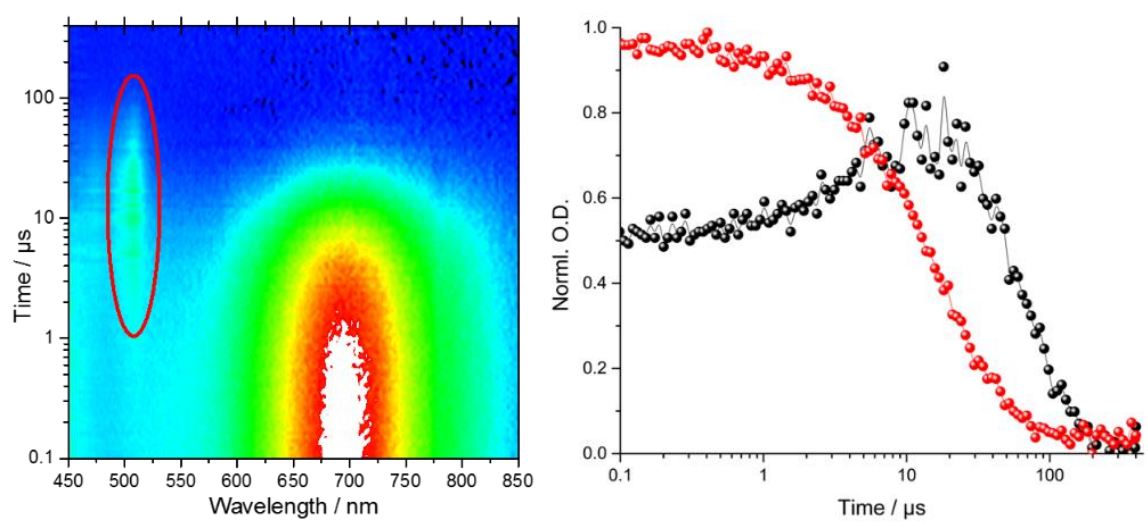

Figure 3. Differential absorption changes (left) obtained upon pump-probe experiments, 480 nm excitation, of $N$-methylfulleropyrrolidine $\left(8.0 \times 10^{-5} \mathrm{M}\right)$ and TIPS-Pn-Me $\left(1.0 \times 10^{-4} \mathrm{M}\right)$ in argon saturated toluene at room temperature with time delays between 0.1 and $400 \mu$ s. The red ellipse highlights the transients of interest. Time absorption profiles (right) of the $\mathrm{N}$ methylfulleropyrrolidine triplet excited-state decay at $700 \mathrm{~nm}$ (red) and the TIPS-Pn-Me triplet excited-state growth and decay at $500 \mathrm{~nm}$ (black).

Independent identification of the triplet excited-state spectra was obtained via triplet-triplet sensitization using $N$-methylfulleropyrrolidine, which has a triplet quantum yield close to unity and a triplet excited-state energy of $1.5 \mathrm{eV}$ (Figure 3). With $N$-methylfulleropyrrolidine as an excited-state donor and (TIPS)2-Pn / TIPS-Pn-Me as excited-state acceptors, selective excitation at $480 \mathrm{~nm}$ coincides with a minimum in the ground-state absorption of pentacene derivatives and gives a triplet excited-state fingerprint at 500-510 nm (Figure 3). By following the temporal evolution at variable concentrations of (TIPS) 2 -Pn and TIPS-Pn-Me, bimolecular triplet-triplet energy transfer rate constants of $(1.6 \pm 0.1) \times 10^{-9} \mathrm{M}^{-1} \mathrm{~s}^{-1}$ were determined. The triplet-triplet sensitization assays also gave lifetimes on the order of $48.2 \pm 2.6 \mu$ s for the triplet excited-state of TIPS-Pn-Me in toluene. 


\section{Photophysical Characterization in Medium and High Concentration Regimes}

$\left(1.0 \times 10^{-3}-1.0 \times 10^{-1} \mathrm{M}\right)$

In the concentration regime from $1.0 \times 10^{-3}$ to $1.0 \times 10^{-1} \mathrm{M}$, absorption assays revealed spectra, which are virtually identical to those recorded at $1.0 \times 10^{-4} \mathrm{M}-$ vide supra. Fluorescence assays, however, turned out to be different (Figures 4 and S12). At first glance, red-shifts of 20 and 30 nm are observed for TIPS-Pn-Me and (TIPS)2-Pn, respectively, upon increasing the concentration up to $1.0 \times 10^{-1}$ M. A closer look reveals that the fluorescence at $649 \mathrm{~nm}$ decreases as the concentration increases and is replaced in the case of (TIPS)2-Pn by a redshifted spectrum that included a long-wavelength maximum at $672 \mathrm{~nm}$ at $1.0 \times 10^{-3} \mathrm{M}$ or at $704 \mathrm{~nm}$ at $1.0 \times 10^{-1}$ M. A qualitatively similar finding is concluded for TIPS-Pn-Me. We observe a red-shift in the spectra from 669 to $686 \mathrm{~nm}$ (Figure 4). Implicit in our observation is the formation of an intermediate state, which takes place only in the excited-state given the lack of changes in the absorption spectra at the different concentrations, as reported in literature (Figures 4 and S12). ${ }^{[27]} \mathrm{A}$ likely rationale is based on the formation of an excimeric state as it resembles those known for excimers. ${ }^{[16,21,33]}$ Notably, its energy of $1.84 \pm 0.05 \mathrm{eV}$ would still be sufficient to support exergonic triplet formation via intermolecular SF. ${ }^{[28]}$
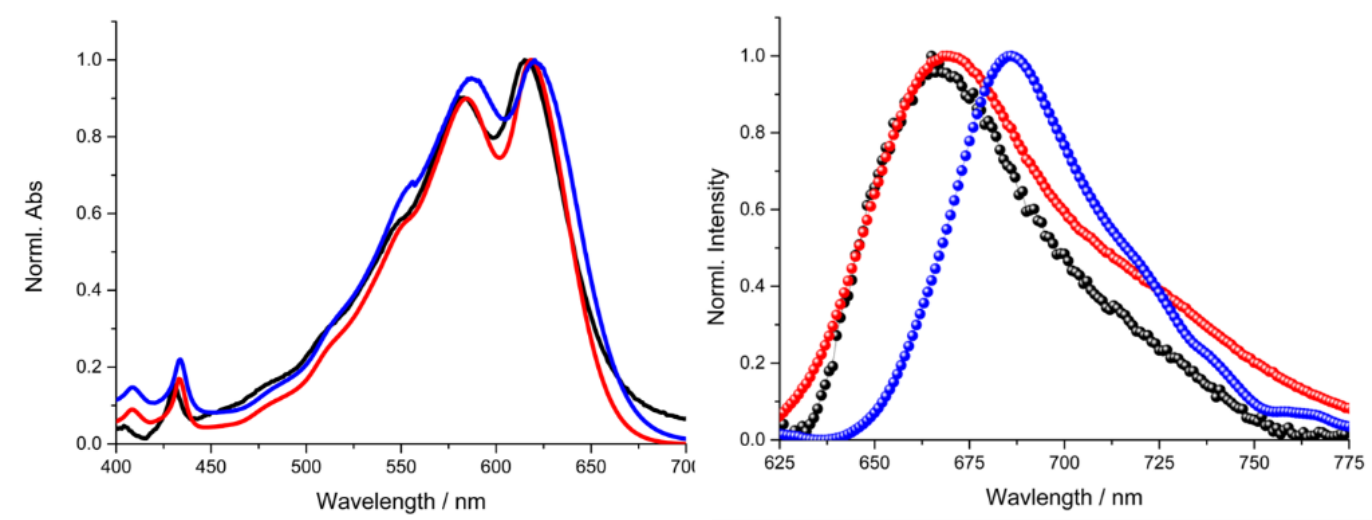

Figure 4. Normalized absorption spectra (left) and normalized fluorescence spectra (right) upon excitation at $530 \mathrm{~nm}$ recorded for variable concentrations of TIPS-Pn-Me in THF: $1.0 \times 10^{-7}$ (black), $1.0 \times 10^{-3}\left(\right.$ red), and $1.0 \times 10^{-1} \mathrm{M}$ (blue) 
Next, fsTA studies were performed for TIPS-Pn-Me and (TIPS)2-Pn at concentrations of $1.0 \times 10^{-3}$ and $1.0 \times 10^{-1} \mathrm{M}$ to investigate intermolecular SF. At $1.0 \times 10^{-3} \mathrm{M}$, signals of the singlet excited-state decay appeared as the end of the $7.5 \mathrm{~ns}$ temporal detection of our set-up is reached (Figure S13). The most notable singlet excited-state features are for TIPS-Pn-Me in the 400-560 nm range with maxima at 445 and $508 \mathrm{~nm}$ and minima at 580 and $642 \mathrm{~nm}$. For (TIPS)2-Pn, the corresponding maxima evolved at 445 and $510 \mathrm{~nm}$ as well as minima at 590 and $640 \mathrm{~nm}$.

In assays with concentrations of $1.0 \times 10^{-1} \mathrm{M}$, emphasis was placed on the competition between the intrinsic singlet excited-state decay and the formation of an intermediate state identified in the fluorescence assays following $530 \mathrm{~nm}$ excitation. ${ }^{[29]}$ At the beginning of the fsTA timescale, that is, after about $500 \mathrm{fs}$, singlet excited-state features evolved, similar to those recorded in the low concentration regime - vide supra. At the end of the fsTA timescale, that is, after $7.5 \mathrm{~ns}$, spectra, which resemble the triplet excited-state of TIPS-Pn-Me or (TIPS)2-Pn are discernible. Importantly, monitoring the singlet excited-state decay and the triplet excited-state growth indicated that the two processes are not directly linked to each other at concentrations of $1.0 \times 10^{-1} \mathrm{M}$. They are, however, indirectly coupled via the population of a transient intermediate. Singlet excited-state decay at, for example, $480 \mathrm{~nm}$ is strictly monoexponential. Excimer intermediate formation takes place in (TIPS) 2 -Pn from a singlet excited-state with a rate constant of $1.1 \times 10^{10} \mathrm{~s}^{-1}$, that is, a lifetime of $90 \pm 0.9$ ps. It reaches its maximum absorption in $770 \pm 8 \mathrm{ps}$, which relates to a rate of $1.3 \times 10^{9} \mathrm{~s}^{-1}$. In the case of TIPS-Pn-Me, a decay of $4.2 \times 10^{11} \mathrm{~s}^{-1}$ is established by deconvolution of the singlet excited-state data. The maximum concentration of the intermediate state is, likewise, reached in $770 \pm 33 \mathrm{ps}$, from which a pseudo first-order decay rate constant of $1.3 \times 10^{9} \mathrm{~s}^{-1}$ was determined. For TIPS-PnMe and (TIPS)2-Pn, the formation of the triplet excited-states sets in at 500-510 nm (Figure 5). 

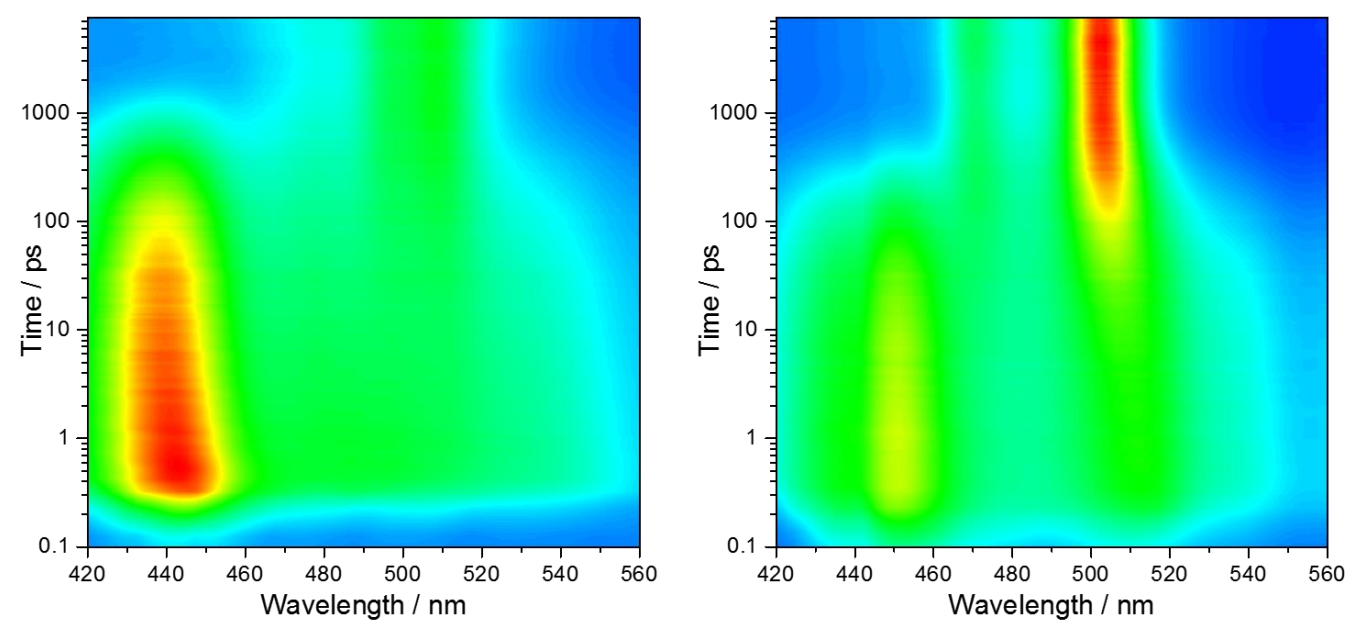

Figure 5. Differential absorption changes of TIPS-Pn-Me (left) and (TIPS)2-Pn (right) in THF at $1.0 \times 10^{-1} \mathrm{M}$ obtained upon pump-probe experiments $(610 \mathrm{~nm})$ at room temperature with time delays between 0.1 and $7.5 \mathrm{~ns}$.

In complementary nsTA experiments, we focused on the maximum triplet excited-state absorptions and their decay for TIPS-Pn-Me (Figure 6) and (TIPS)2-Pn (Figure S15). In line with the fsTA experiments, the singlet excited-states can be deconvoluted with our nsTA resolution in concentrations of $1.0 \times 10^{-1} \mathrm{M}$. Likewise, we note the intermediate state for TIPSPn-Me and (TIPS)2-Pn as it transforms into the corresponding triplet excited-states, which was deconvoluted via nsTA. In the case of TIPS-Pn-Me, the intermediate state gives rise to a maximum at $500-510 \mathrm{~nm}$, followed by minima at 580 and $642 \mathrm{~nm}$. For (TIPS)2-Pn, characteristic fingerprints for the triplet excited-state include a maximum at $510 \mathrm{~nm}$ as well as minima at 590 and $642 \mathrm{~nm}$.

Singlet excited-state deactivation of (TIPS)2-Pn, TIPS-Pn-Me, and TIPS-Pn-H was investigated at $1.0 \times 10^{-3}$ and $1.0 \times 10^{-2} \mathrm{M}$. At $1.0 \times 10^{-3} \mathrm{M}$, singlet excited-state lifetimes of $16.0 \pm 0.1,5.1 \pm 0.1$, and $11.0 \pm 0.1 \mathrm{~ns}$ relate to mono-exponential decay rates between 
$0.63 \times 10^{8}$ and $1.96 \times 10^{8} \mathrm{~s}^{-1} .{ }^{1}$ At $1.0 \times 10^{-2} \mathrm{M}$, the singlet excited-state decay are strictly monoexponential with lifetimes of $8.3 \pm 0.1,11.0 \pm 0.1$ and $4.8 \pm 0.5$ ns for (TIPS)2-Pn, TIPS-PnMe and TIPS-Pn-H, respectively, and rates between $2.08 \times 10^{8}$ and $0.91 \times 10^{8} \mathrm{~s}^{-1}$ as determined by deconvolution. As mentioned above, the change in singlet excited-state lifetime is a first sign of intermolecular SF (Figure 6). The underlying singlet excited-state quenching is seen to correlate with a faster and more efficient formation of the intermediate state and, subsequently, of the triplet excited-state. ${ }^{[30]}$

Triplet excited-state deactivations of (TIPS)2-Pn, TIPS-Pn-Me, and TIPS-Pn-H were likewise investigated at a concentration of $1.0 \times 10^{-3} \mathrm{M}$ and featured lifetimes of $31.0 \pm 0.7,61.0 \pm 3$, and $1.3 \pm 0.1 \mu \mathrm{s}$, respectively. At $1.0 \times 10^{-1} \mathrm{M}$, the triplet excited-state undergoes transformation to several intermediates - vide supra - and the shorter lifetimes for the excitedstate deactivation are $6.2 \pm 0.4 \mu \mathrm{s}, 11.0 \pm 0.1 \mu \mathrm{s}$ and $57.0 \pm 0.2 \mathrm{~ns}$ for (TIPS) 2 -Pn, TIPS-PnMe and TIPS-Pn-H, respectively.
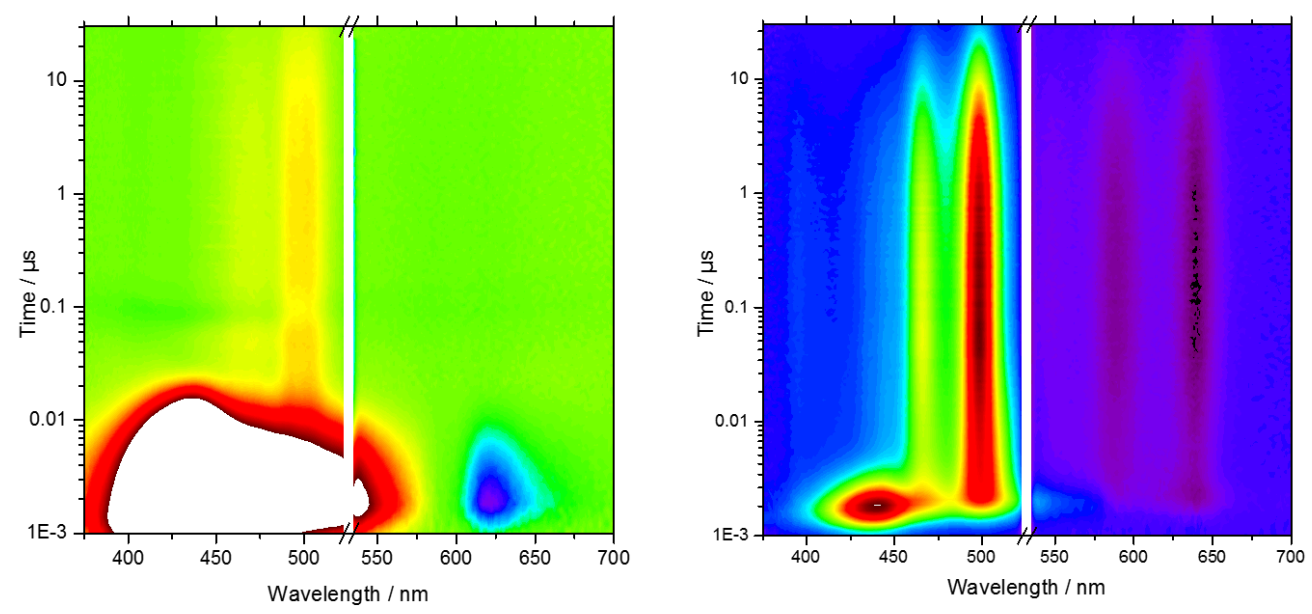

\footnotetext{
${ }^{1}$ For concentrations of $1.0 \times 10^{-2} \mathrm{M}$, slightly more rapid singlet excited-state deactivations prompt towards a different pathway as it becomes available.
} 
Figure 6. Differential absorption changes (visible) obtained upon pump-probe experiments $(532 \mathrm{~nm})$ at room temperature with time delays between 0 and $35 \mu$ s of TIPS-Pn-Me in THF at $1.0 \times 10^{-3} \mathrm{M}($ left $)$, and $1.0 \times 10^{-1} \mathrm{M}$ (right).

To corroborate intermolecular SF, the triplet excited-state population must be determined. Technical limitations hinder, however, reliable quantum yield calculations. In the aforementioned assays, a clear relationship between the normalized singlet excited-state absorption maxima and absorption maxima of the triplet excited-state reflects the overall population of the respective states. For (TIPS)2-Pn and TIPS-Pn-Me, a congruent behavior is found in the absorption of the triplet excited-state signals, while the behavior for TIPS-Pn-H is different (Figure 7). The first two exhibit SF behavior, with an excimer-intermediate, whereas the last one does not. In particular, a steady increase in the absorption maxima from 0.29-0.34 a.u. is noted in the triplet excited-state spectrum for TIPS-Pn-Me and also a notable increase is determined for (TIPS) 2-Pn with absorptions ranging from 0.34 to 0.53 a.u. Furthermore, both TIPS-Pn-Me and (TIPS)2-Pn show maximum ratios between 1.28-1.33 a.u. in the higher concentration assays (Figure 7 and S18). The increase in the triplet excited-state absorption prompts to a larger population of the triplet excited-state relative to the singlet excited-state and provides a solid basis for SF.

In the case of TIPS-Pn-H, there is also an increase of the relative absorption intensity from 0 to 0.36 a.u. However, this is more likely due to a photo-induced cyclization, rather than SF. ${ }^{[34]}$ In particular, the unique structure of TIPS-Pn-H, lacking substitution in the 13-position, renders it susceptible to reactions in the excited-state, such as formation of dimer 2 (Figures 2 and S17). ${ }^{[26,27,29]}$ 

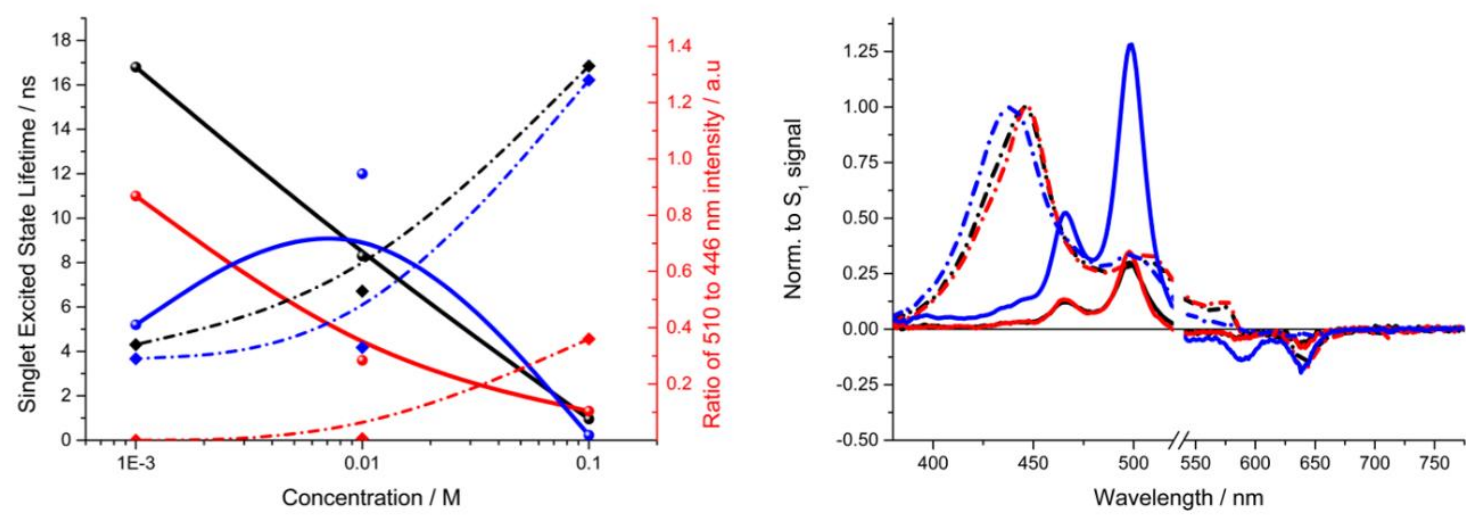

Figure 7. (Left) Singlet excited-state lifetime (line) on the left axis and ratio of relative 510 to $446 \mathrm{~nm}$ intensity (dash) on the right axis as a function of concentration for (TIPS)2-Pn (black), TIPS-Pn-Me (blue), and TIPS-Pn-H (red). (Right) Normalized spectra of the singlet excitedstate (dash) compared to the absorption maxima of the triplet excited-state (line) for TIPS-PnMe at $1.0 \times 10^{-3}$ (black), $1.0 \times 10^{-2}\left(\right.$ red), and $1.0 \times 10^{-1}$ (blue) M for nsTA assays.

\section{Global Analysis}

The implementation of multiwavelength modeling ${ }^{[38]}$ for fs- and nsTA kinetics has provided valuable insight into the study of intra- and intermolecular SF. ${ }^{[39]}$ Using this methodology, a key step in exergonic SF with pentacene derivatives is established (Figure 8). Namely, the formation of a transient excimer state is identified, and the decay of this state is linked to the triplet excited-state formation at $500-510 \mathrm{~nm}$. 


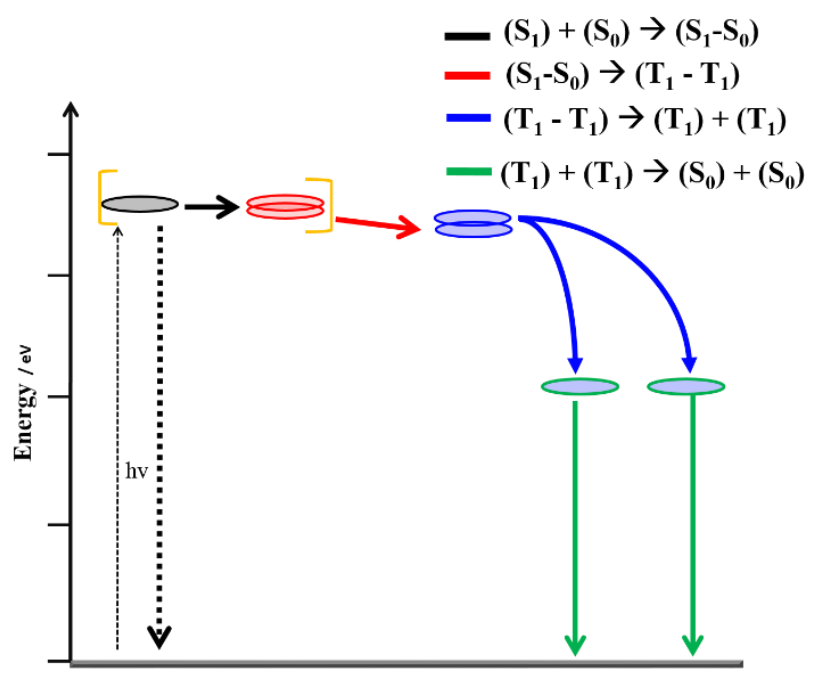

Figure 8. Schematic illustration of the global analysis model for fs- and nsTA assays for (TIPS)2-Pn and TIPS-Pn-Me at $1.0 \times 10^{-1} \mathrm{M}$. The dynamics in the orange parenthesis can be followed via fsTA, whereas other processes are established from deconvolution of the nsTA assays (ovals represent schematic depiction of pentacene chromophores).

On one hand, our approach includes the investigation of the dynamics for excimer formation and its transformation to the correlated triplet excited-state. On the other hand, diffusional splitting into independent triplet excited-states, and recovery of the ground state is explored. Our recent results on intramolecular SF in pentacene dimers combined with diffusion controlled reaction schemes were instrumental for the development of a suitable sequential model for global analysis. ${ }^{[39]}$ 

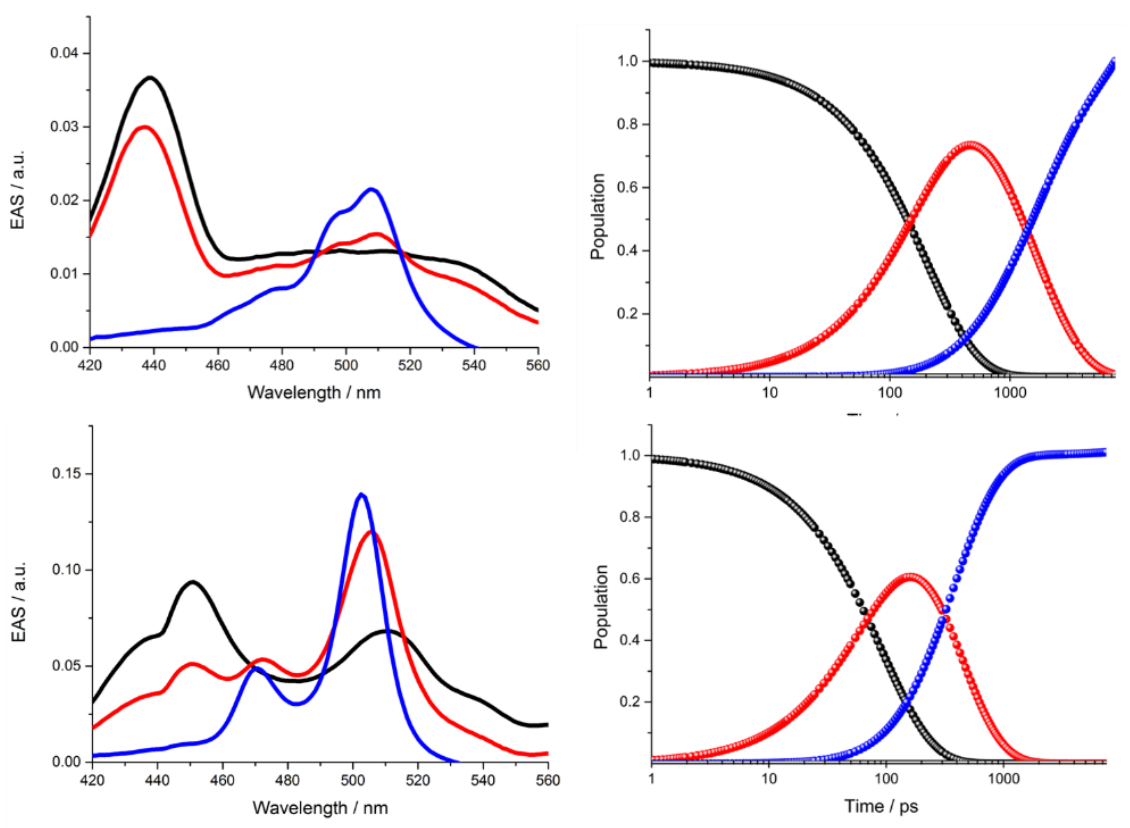

Figure 9. Global analysis of TIPS-Pn-Me (top) and (TIPS)2-Pn (bottom) in THF at $1.0 \times 10^{-1}$ $\mathrm{M}$ at room temperature with time delays between 0 and $7.5 \mathrm{~ns}$. Evolution-associated transient absorption spectra (left) in the visible range $(420-560 \mathrm{~nm})$ of the deconvoluted species as shown in the model in Figure 8: $\mathrm{S}_{1}$ (black), $\left(\mathrm{S}_{1}-\mathrm{S}_{0}\right)(\mathrm{red})$, and $\left(\mathrm{T}_{1}-\mathrm{T}_{1}\right)$ (blue). Population kinetics (right) for each of the determined species in their corresponding colors with collection times from $1 \mathrm{ps}$ to $7.5 \mathrm{~ns}$.

To unravel the kinetics of intermolecular SF in the high concentration regime, we applied a sequential global analysis (Figure 9). On the fs- and nsTA timescales, a three-state model gives the best fit to the results. In detail, the singlet excited-state $\left(\mathrm{S}_{1}\right)$ was resolved as the first transient in fs- and nsTA assays. $\left(\mathrm{S}_{1}\right)$ decays and yields a transient $\left(\mathrm{S}_{1}-\mathrm{S}_{0}\right)$ as the second transient by interacting with a neighboring molecule in its ground state $\left(\mathrm{S}_{0}\right)$. This mechanism seems to accurately describe measurements between $1.0 \times 10^{-2}$ and $1.0 \times 10^{-1} \mathrm{M}$ for TIPS-Pn-Me and (TIPS) 2 -Pn. At the highest concentration, $\left(\mathrm{S}_{1}-\mathrm{S}_{0}\right)$ is replaced by the third transient, namely the coupled triplet excited-state $\left(\mathrm{T}_{1}-\mathrm{T}_{1}\right)$. At $1.0 \times 10^{-2} \mathrm{M},\left(\mathrm{S}_{1}-\mathrm{S}_{0}\right)$ for TIPS-Pn-Me and (TIPS) $)_{2}$ Pn have similar lifetimes, between 25-30 ns. T At higher concentrations, more rapid evolution, 
with lifetimes in the order of $770 \mathrm{ps}$, is further evidence for the efficient and favored formation of the $\left(T_{1}-T_{1}\right)$ state (Figure 9$)$.

Subsequently, we developed a model for fitting the nsTA results, based on three transients (Table 1 and Figure 10). Considering the resolution of our experimental set-up, the first transient is assigned as $\left(\mathrm{S}_{1}-\mathrm{S}_{0}\right)$, the same as postulated in the fsTA model (Figure 9) for high concentration assays. The $\left(\mathrm{S}_{1}-\mathrm{S}_{0}\right)$ then transforms into the second transient, a coupled pair of triplet excited-states $\left(\mathrm{T}_{1}-\mathrm{T}_{1}\right)$. For (TIPS) 2 -Pn and TIPS-Pn-Me, the diffusional split takes place with lifetimes of $13.0 \pm 0.5$ and $15 \pm 0.2 \mathrm{~ns}$ and rates of $6.6 \pm 0.1$ and $7.8 \pm 0.1 \times 10^{7} \mathrm{~s}^{-1}$, respectively. In the third and final step, free triplet excited-states $\left(\left(T_{1}\right)+\left(T_{1}\right)\right)$ decay to the ground state with triplet excited-state lifetimes of $6.2 \pm 0.7$ and $11 \pm 0.1 \mu \mathrm{s}$, respectively. Thereby, $\left(\left(\mathrm{T}_{1}\right)+\left(\mathrm{T}_{1}\right)\right)$ could decay by means of interacting with a ground state $\left(\mathrm{S}_{0}\right)$ or a triplet excited-state $\left(T_{1}\right)$, or via unimolecular deactivation. It is noted that the interaction with $\left(T_{1}\right)$ is mainly observed in the high concentration regime, where the SF triplet excited-state population is higher.

Table 1. Lifetimes (s) obtained through data deconvolution for TIPS-Pn-Me and (TIPS)2-Pn in $\mathrm{THF}$ at $1.0 \times 10^{-1} \mathrm{M}$ at room temperature.

\begin{tabular}{l|cc|cc}
\hline & $\left(\mathbf{S}_{\mathbf{1}}\right)+\left(\mathbf{S}_{\mathbf{0}}\right) \rightarrow\left(\mathbf{S}_{\mathbf{1}}-\mathbf{S}_{\mathbf{0}}\right)^{\mathbf{a}, \mathbf{c}}$ & $\left(\mathbf{S}_{\mathbf{1}}-\mathbf{S}_{\mathbf{0}}\right) \rightarrow\left(\mathbf{T}_{\mathbf{1}}-\mathbf{T}_{\mathbf{1}}\right)^{\mathbf{a}, \mathbf{b}}$ & $\begin{array}{c}\left(\mathbf{T}_{\mathbf{1}}-\mathbf{T}_{\mathbf{1}}\right) \rightarrow\left(\left(\mathbf{T}_{\mathbf{1}}\right)+\right. \\
\left.\left(\mathbf{T}_{\mathbf{1}}\right)\right)^{\mathbf{b}}\end{array}$ & $\left(\left(\mathbf{T}_{\mathbf{1}}\right)+(\mathbf{T} \mathbf{1})\right)^{\mathbf{b}} \rightarrow\left(\mathbf{S}_{\mathbf{0}}\right)+\left(\mathbf{S}_{\mathbf{0}}\right)$ \\
\hline TIPS-Pn-Me & $2.4 \times 10^{-12}$ & $7.7 \times 10^{-10}$ & $1.5 \times 10^{-8}$ & $1.1 \times 10^{-5}$ \\
\hline (TIPS)2-Pn & $9.0 \times 10^{-11}$ & $7.7 \times 10^{-10}$ & $1.3 \times 10^{-8}$ & $6.2 \times 10^{-6}$ \\
\hline
\end{tabular}

\footnotetext{
a) Data for $\left(\mathrm{S}_{1}\right)$ and $\left(\mathrm{S}_{1}-\mathrm{S}_{0}\right)$ were determined using the fsTA model.

b) Data for $\left(\mathrm{S}_{1}-\mathrm{S}_{0}\right),\left(\mathrm{T}_{1}-\mathrm{T}_{1}\right)$, and $\left(\mathrm{T}_{1}\right)+\left(\mathrm{T}_{1}\right) \rightarrow\left(\mathrm{S}_{0}\right)+\left(\mathrm{S}_{0}\right)$ were determined using the nsTA model.
} 

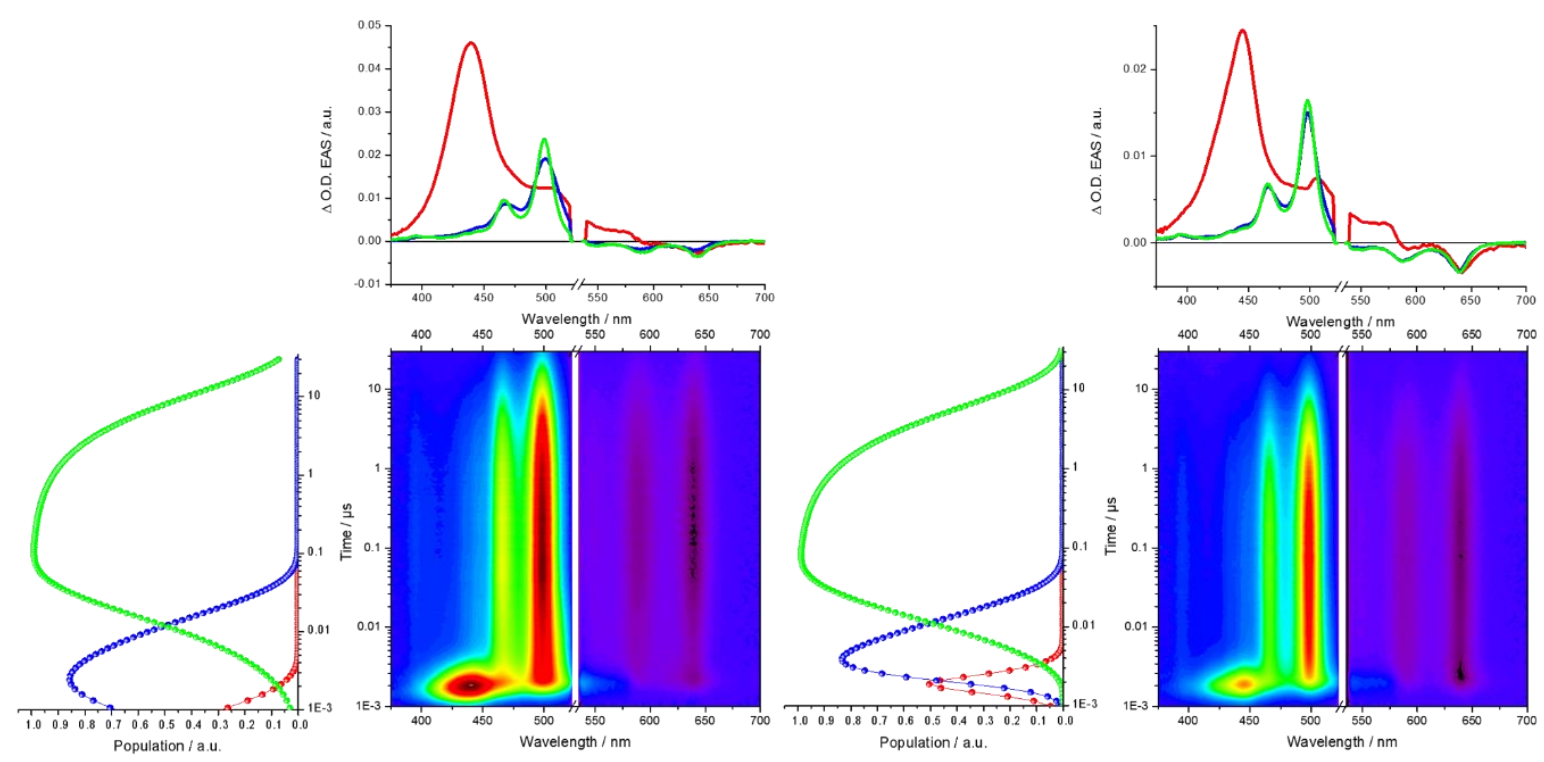

Figure 10. Global analysis of nsTA data of TIPS-Pn-Me (left) and (TIPS)2-Pn (right) in THF at $1.0 \times 10^{-1} \mathrm{M}$. Spectra of excited-state species (top) in the visible range (375-700 $\mathrm{nm}$ ) of the deconvoluted species as shown in the model in Figure 8. Population kinetics (bottom, left) for each species, with colors respective to the model presented in Figure 8. Differential absorption changes (bottom, right) obtained upon pump-probe experiments $(530 \mathrm{~nm})$ at room temperature with time delays between 0 and $35 \mu$ s.

The more rapid deactivation of the singlet excited-state upon increased concentration is not surprising, since collision of a molecule in the singlet excited-state with another in the ground state is the first step of the intermolecular pathway to SF. Therefore, formation of the $\left(\mathrm{S}_{1}-\mathrm{S}_{0}\right)$ state is concentration dependent in the range from $1.0 \times 10^{-3}$ to $1.0 \times 10^{-1} \mathrm{M}$. The $\left(\mathrm{T}_{1}-\mathrm{T}_{1}\right)$ state formation is unlikely to be dependent on the concentration, the diffusion splitting in the case of TIPS-Pn-Me takes place at $15 \pm 0.8 \mathrm{~ns}$ and decay to $\mathrm{S}_{0}$ in $11 \pm 0.3 \mu \mathrm{s}$, hence, experimental decay of $6.6 \pm 0.2 \times 10^{7}$ and $8.6 \pm 1.0 \times 10^{4} \mathrm{~s}^{-1}$, respectively.

The recovery of the ground state could occur by unimolecular deactivation to $\left(\mathrm{S}_{0}\right)$ or via annihilation with another TIPS-Pn-Me molecule in either $\left(\mathrm{S}_{0}\right)$ or $\left(\mathrm{T}_{1}\right)$ state, processes that are 
strongly concentration dependent. Triplet-triplet annihilation is thus only meaningful in the regime of high SF quantum yield, but, it is typically outperformed by collisional deactivation with a TIPS-Pn-Me molecule in $\left(\mathrm{S}_{0}\right)$.

Finally, a comparison of singlet excited-state lifetimes and triplet excited-state populations between (TIPS) 2 -Pn and TIPS-Pn-Me is instructive. Namely, the $\left(S_{1}\right)$ lifetime is affected by the degree of steric protection of the pentacene core by the appended functional group. At a concentration of $1.0 \times 10^{-1} \mathrm{M}$, the lifetime of TIPS-Pn-Me is less than $5 \mathrm{ps}$ and, in turn, leads to a faster transition to an "excimer" state and electronic coupling between TIPS-Pn-Me. In our model, all other states, with the exception of $\left(\left(\mathrm{T}_{1}\right)+\left(\mathrm{T}_{1}\right)\right)$, proceed with nearly identical dynamics in both (TIPS)2-Pn and TIPS-Pn-Me. Lifetimes and rates, which are only detected for (TIPS) 2 -Pn and TIPS-Pn-Me, are assigned as direct effects of functional groups at the 6and 13-positions of the pentacene and are in line with the lack of triplet excited-states in TIPSPn-H. The fate of TIPS-Pn-H is likely a result of the unprotected pentacene moiety that facilitates competing quenching mechanisms such as cyclization reactions or oxidative degradation processes. In the absence of steric shielding, the pentacene skeleton is chemicallyreactive and unstable for the required strong and stable, electronic coupling. ${ }^{[34,40-45]}$

\section{Theoretical and Computational Analysis}

For the theoretical characterization of SF in the systems investigated, we have used different conformations of (TIPS)2-Pn, TIPS-Pn-Me, and TIPS-Pn-H obtained from the corresponding X-ray crystallographic data as reference structures (Figure 11). For high concentrations, this choice is sensible as it can be expected that these geometrical arrangements will be preferred over other less stable configurations under appropriate experimental conditions.

To reduce the computational cost, the $i \mathrm{Pr}_{3} \mathrm{Si}$-groups have been modeled using $\mathrm{H}_{3} \mathrm{Si}$-groups. The different electronic states involved in the SF mechanism have been characterized in both the adiabatic and diabatic representations for all systems investigated. All the calculations were 
carried out using the extended multiconfiguration quasi-degenerate perturbation theory (XMCQDPT) approach $^{[46]}$ and a double $\zeta$-basis set (see SI). ${ }^{[47]}$ In the calculations, solvent effects have not explicitly been accounted for. However, previous works have shown that in vacuo calculations can provide a qualitatively correct description of the SF process, in particular when nonpolar solvent are used. ${ }^{[16,39,48,49]}$

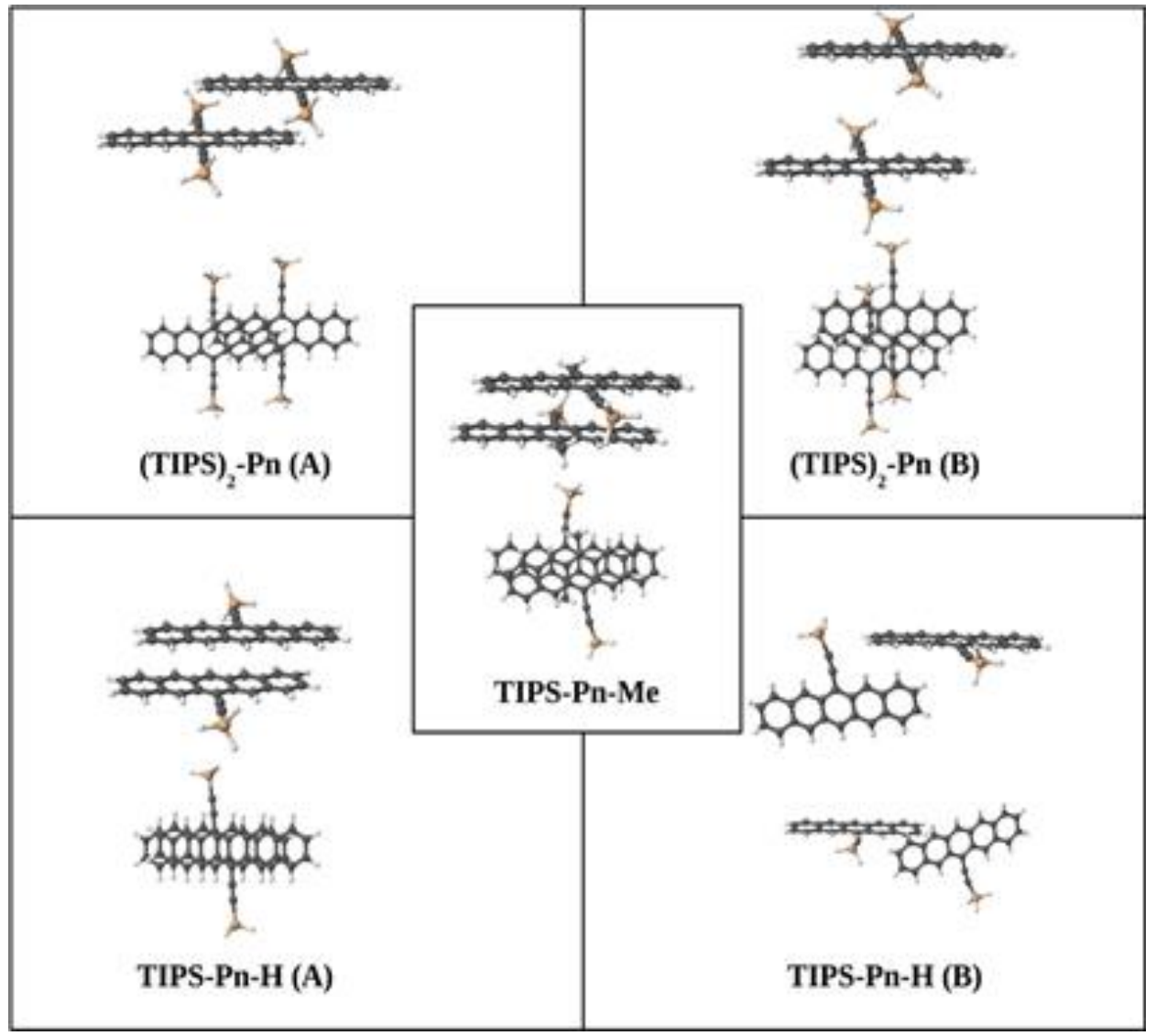

Figure 11. Geometrical arrangement of the pentacene dimers investigated based on crystallographic analyses - Figures S1 and S2.

Our results show good agreement between the vertical excitation energies calculated for the lowest-lying bright states of (TIPS)2-Pn, TIPS-Pn-Me, and TIPS-Pn-H and the experimental absorption maxima (Table S1). In particular, the vertical excitation energy of the lowest-lying bright state of (TIPS)2-Pn in both the (A) and (B) conformations, 1.82 and $2.01 \mathrm{eV}$, respectively, agrees well with the experimental value found for the absorption maximum, $1.94 \mathrm{eV}$. Similar 
agreement is found for the absorbing states of both conformations of TIPS-Pn-H (Table S1), which are in line with the experimental value $2.03 \mathrm{eV}$. Finally, the vertical excitation energy of $1.98 \mathrm{eV}$ for the absorbing state of TIPS-Pn-Me corresponds well with that of determined from experiment, $2.03 \mathrm{eV}$. Concerning the distribution of the different low-lying electronic states, the multi-excitonic (ME)-like and the locally excited (LE)-like states are almost degenerate in all systems, with energy differences of $\sim 0.1 \mathrm{eV}$. On the other hand, the order and relative energies of the doubly excited (DE) and charge-transfer (CT) states are more sensitive to changes in the relative geometrical arrangement of the monomers in the dimer and differ significantly from system to system (Table S1).

We have calculated, and present herein in a simplified notation, the relevant diabatic electronic states and the corresponding electronic couplings for the conformations under consideration, in order to better understand the mechanism of SF in these systems. This analysis was made using Truhlar's four-fold-way diabatization method. ${ }^{[50,51]}$ The diabatic basis set employed includes a) the ground state $\left.\left(\mathrm{S}_{0}-\mathrm{S}_{0}\right), \mathrm{b}\right)$ the correlated triplet pair (or ME) state $\left(\mathrm{T}_{1}-\mathrm{T}_{1}\right)$ in which a triplet electronic state is localized in each monomer and coupled to form a singlet electronic state, c) the locally excited (LE) states $\left(\mathrm{S}_{1}-\mathrm{S}_{0}\right)$ and $\left(\mathrm{S}_{0}-\mathrm{S}_{1}\right)$ in which the excitation is localized in one of the monomers, and d) the $\mathrm{CT}$ states $(\mathrm{CA})$ and $(\mathrm{AC})$ where $\mathrm{C}$ and $\mathrm{A}$ stand for the one-electron oxidized radical cation and the one-electron reduced radical anion form of the pentacene derivatives, respectively. Because of energy considerations, two doubly excited diabatic states $(\mathrm{D}-\mathrm{E})_{1}$ and $(\mathrm{D}-\mathrm{E})_{2}$ were also included. ${ }^{[5,6,34,52]}$

Tables S2-S4 collect the diabatic electronic Hamiltonian for the systems investigated. In all systems, the electronic coupling of the multiexcitonic $\left(\mathrm{T}_{1}-\mathrm{T}_{1}\right)$ state with the $\mathrm{LE}\left(\mathrm{S}_{1}-\mathrm{S}_{0}\right)$ and $\left(\mathrm{S}_{0^{-}}\right.$ $\mathrm{S}_{1}$ ) states is small, reaching a maximum value of $\sim 9 \mathrm{meV}$ for TIPS-Pn-Me. The electronic coupling of the CT states, $(\mathrm{C}-\mathrm{A})$ and $(\mathrm{A}-\mathrm{C})$, with the $\left(\mathrm{T}_{1}-\mathrm{T}_{1}\right)$ state exhibits more complex behavior. Specifically, these couplings show a clear dependence on the relative orientation of 
the monomers as well as the intermolecular distance between them. Thus, the largest couplings are found for the dimers that are characterized by the shortest intermolecular distances and in which the monomers are slipped-stacked ((TIPS)2-Pn (A)) or partially staggered (TIPS-PnMe). In contrast, dimers with the largest intermolecular distances [(TIPS)2-Pn (B) and TIPSPn-H (B)] exhibit the weakest coupling. In addition, the relative energies of $(\mathrm{C}-\mathrm{A})$ and $(\mathrm{A}-\mathrm{C})$ with respect to $\left(T_{1}-T_{1}\right),\left(S_{1}-S_{0}\right)$, and $\left(S_{0}-S_{1}\right)$ also increase due to the smaller contribution of the Coulomb interaction between $\mathrm{C}$ and A to the total energy of the CT states.

To investigate the impact that conformation has on the SF mechanism and to assess the role of CT states in the process, we have quantified the contribution of the direct and mediated mechanism calculating the effective SF coupling, $\boldsymbol{V}_{\boldsymbol{e f f}}$, of $\left(\mathrm{S}_{1-} \mathrm{S}_{0}\right)$ to $\left(\mathrm{T}_{1}-\mathrm{T}_{1}\right)$ or $\left(\mathrm{S}_{0}-\mathrm{S}_{1}\right)$ to $\left(\mathrm{T}_{1}\right.$ $\mathrm{T}_{1}$ ) - equation $\mathrm{S} 1$, see Supporting Information for details. ${ }^{[53,54]}$ The results obtained are collected in Table S5 and show that dimeric pairs with the largest intermolecular distance and the smallest $\pi$ overlap density ((TIPS)2-Pn (B) and TIPS-Pn-H (B)) have negligible direct, mediated mechanisms, and, therefore, effective SF couplings for both LE states. The remaining dimers, characterized by shorter intermolecular distances, have non-negligible effective couplings, with the main contribution arising from the mediated mechanism. These results are consistent with a superexchange-like mechanism, in which the CT states act as virtual states in the SF process. ${ }^{[6,54,55]}$ Considering that the CT states in these systems do not lie too high in energy with respect to the LE states $(<0.4 \mathrm{eV}$, see Table S5), possible transient population of the CT states could eventually take place in polar solvents or if the neighboring chromophores are sufficiently close to stabilize the CT states with respect to the LE states.

\section{Conclusions}

In the current work, we have explored three pentacene derivatives within the context of intermolecular SF by means of advanced photophysical measurements, global analysis modeling, as well as ab-initio calculations. We have demonstrated that the triplet excited-state 
population and deactivation are affected through variation of the functional groups appended to the acene core. Key to the differences is the rate of diffusional encounter between one pentacene in its singlet excited-state and another in its ground state, which gives rise to excimer characteristics. Our work corroborates earlier work on pentacene derivatives, for which SF is sufficiently exergonic, but contrasts work on slightly endergonic SF in tetracene derivatives. Specific mechanistic conclusions are based on spectroscopic analyses of chromophore concentrations ranging from as low as $1.0 \times 10^{-3}$ to as high as $1.0 \times 10^{-1} \mathrm{M}$ and corroborate SF under the conditions of our experiments. On the other hand, the susceptibility of the pentacene moiety to undergo photo-induced cyclization at the 6-and 13-positions is known in literature and becomes problematic in more concentrated solutions. For optimization of SF, it is thus advantageous to incorporate substituents at the 6-and 13-positions to stabilize the pentacene chromophore through preventing in situ reactivity. This is important for future modification of acenes for energy conversion schemes, as previously noted. ${ }^{[56-58]}$

Based on fluorescence assays, a newly developing species in the high-concentration regime is of great relevance. The observed species resembles that of an excimer-like state, whose existence is independently confirmed in fsTA assays and indicates that the exothermic SF involves a diffusive, excimeric intermediate state. Theoretical calculations suggest a strong coupling between the singlet excited-state and a CT state, which results in the efficient SF. This singlet excited-state transforms into the non-emitting triplet excited-state $\left(T_{1}-T_{1}\right)$ featuring an overall spin multiplicity of one. In complementary nsTA assays, we corroborated that the nonemitting, triplet excited-states, which are strongly coupled and entangled, undergo processes that result in a pair of triplet excited-states. Ultimately, a pair of independent triplet excitedstates $\left(\left(\mathrm{T}_{1}\right)+\left(\mathrm{T}_{1}\right)\right)$ is formed. In the final step, namely recovery of the ground state, annihilation via interaction with a ground-state or a triplet excited-state species dominates. 
With respect to the impact that structural factors may have on the SF mechanism, the high-level multi-reference perturbation theory calculations carried out in this work show that the direct coupling is small. Furthermore, our calculations show a correlation of the electronic coupling of the ME and LE states with the relative orientation and distance between the monomers. Specifically, the largest coupling is found for those dimer structures with the shortest intermolecular distances and in which the monomers are distorted from a stacked-like conformation to with a slipped-stacked or to a partially staggered arrangement. As a result, these calculations point to a mediated superexchange-like mechanism as the main reaction channel for SF, which lends itself to external control using a suitable polar solvent or to the use of substituent groups with different degrees of bulkiness and/or steric hindrance.

It is important to remark that not all the pentacene derivatives examined in this study undergo SF as photodegradation sets in. This finding highlights the importance of strategic synthetic functionalization with groups as small as a methyl moiety can enable SF and avoid photodegradation. ${ }^{[58]}$, We are currently probing intermolecular SF with solid-state samples of the various pentacene derivatives, with an emphasis on the crystal packing as a means to control the dynamics and the yield of SF.

CCDC 1815873 (ketone 1), 1812258 (TIPS-Pn-Me), 1839937 (endoperoxide S2), and 1815872 (TIPS-Pn-H) contain the supplementary crystallographic data for this paper. These data can be obtained free of charge from The Cambridge Crystallographic Data Centre via www.ccdc.cam.ac.uk/data_request/cif. Further details of the crystal structure investigation(s) may be obtained by contacting the author (RRT)

\section{Supporting Information}

Supporting Information is available from the Wiley Online Library or from the authors. 


\section{Acknowledgements}

Generous allocation of computing time at the computing centers in Erlangen (Regionales Rechenzentrum Erlangen), Munich (Leibniz Rechenzentrum der Bayerischen Akademie der Wissenschaften in München) and Jülich (Jülich Supercomputing Center) is gratefully acknowledged. Funding is gratefully acknowledged from the Emerging Fields Initiative (EFI) "Singlet Fission" supported form the Friedrich-Alexander Universität Erlangen-Nürnberg, as well as the Natural Sciences and Engineering Research Council of Canada (NSERC), the Cluster for Excellence Engineering of Advanced Materials (EAM) and "Solar Technologies Go Hybrid" - an initiative of the Bavarian State Ministry for Science, Research and Art. PBC acknowledges financial support from DFG (DFG-CO-167/1-1), the Regional Council for Industry and Employment of the Principality of Asturias, and the European Regional Development Fund (ERDF). We acknowledge Dr. Dan Lehnherr for the initial synthesis of TIPS-Pn-Me.

Received: ((will be filled in by the editorial staff))

Revised: ((will be filled in by the editorial staff))

Published online: ((will be filled in by the editorial staff)) 


\section{References}

[1] P. J. Jadhav, A. Mohanty, J. Sussman, J. Lee, M. A. Baldo, Nano Lett. 2011, 11, 1495.

[2] L. Yang, M. Tabachnyk, S. L. Bayliss, M. L. Böhm, K. Broch, N. C. Greenham, R. H. Friend, B. Ehrler, Nano Lett. 2015, 15, 354.

[3] P. D. Reusswig, D. N. Congreve, N. J. Thompson, M. A. Baldo, Appl. Phys. Lett. 2012, 101, 113304.

[4] D. N. Congreve, J. Lee, N. J. Thompson, E. Hontz, S. R. Yost, P. D. Reusswig, M. E. Bahlke, S. Reineke, T. Van Voorhis, M. A. Baldo, Science. 2013, 340, 334.

[5] M. B. Smith, J. Michl, Chem. Rev. 2010, 110, 6891.

[6] M. B. Smith, J. Michl, Annu. Rev. Phys. Chem. 2013, 64, 361.

[7] P. M. Zimmerman, C. B. Musgrave, M. Head-Gordon, Acc. Chem. Res. 2013, 46, 1339.

[8] S. R. Yost, J. Lee, M. W. B. Wilson, T. Wu, D. P. McMahon, R. R. Parkhurst, N. J. Thompson, D. N. Congreve, A. Rao, K. Johnson, M. Y. Sfeir, M. G. Bawendi, T. M. Swager, R. H. Friend, M. A. Baldo, T. Van Voorhis, Nat. Chem. 2014, 6, 492.

[9] N. Monahan, X.-Y. Zhu, Annu. Rev. Phys. Chem. 2015, 66, 601.

[10] A. J. Musser, M. Maiuri, D. Brida, G. Cerullo, R. H. Friend, J. Clark, J. Am. Chem. Soc. 2015, 137, 5130 .

[11] H. Tamura, M. Huix-Rotllant, I. Burghardt, Y. Olivier, D. Beljonne, Phys. Rev. Lett. 2015, 115, 107401.

[12] S. L. Bayliss, A. D. Chepelianskii, A. Sepe, B. J. Walker, B. Ehrler, M. J. Bruzek, J. E. Anthony, N. C. Greenham, Phys. Rev. Lett. 2014, 112, 238701.

[13] C. J. Bardeen, Annu. Rev. Phys. Chem. 2014, 65, 127.

[14] S. T. Roberts, R. E. McAnally, J. N. Mastron, D. H. Webber, M. T. Whited, R. L. Brutchey, M. E. Thompson, S. E. Bradforth, J. Am. Chem. Soc. 2012, 134, 6388.

[15] H. Marciniak, I. Pugliesi, B. Nickel, S. Lochbrunner, Phys. Rev. B 2009, 79, 235318.

[16] B. J. Walker, A. J. Musser, D. Beljonne, R. H. Friend, Nat. Chem. 2013, 5, 1019.

[17] H. L. Stern, A. J. Musser, S. Gelinas, P. Parkinson, L. M. Herz, M. J. Bruzek, J. Anthony, R. H. Friend, B. J. Walker, Proc. Natl. Acad. Sci. U. S. A. 2015, 112, 7656.

[18] A. J. Musser, M. Al-Hashimi, M. Maiuri, D. Brida, M. Heeney, G. Cerullo, R. H. Friend, J. Clark, J. Am. Chem. Soc. 2013, 135, 12747.

[19] Y. Kasai, Y. Tamai, H. Ohkita, H. Benten, S. Ito, J. Am. Chem. Soc. 2015, 137, 15980.

[20] J. Zirzlmeier, D. Lehnherr, P. B. Coto, E. T. Chernick, R. Casillas, B. S. Basel, M. Thoss, R. R. Tykwinski, D. M. Guldi, Proc. Natl. Acad. Sci. U. S. A. 2015, 112, 5325.

[21] A. Rao, R. H. Friend, Nat. Rev. 2017, $2,1$.

[22] C. Hetzer, D. M. Guldi, R. R. Tykwinski, Chem. - Eur. J. 2018, 24, 8245.

[23] A. Japahuge, T. Zeng, ChemPlusChem 2018, 83, 146.

[24] R. D. Pensack, E. E. Ostroumov, A. J. Tilley, S. Mazza, C. Grieco, K. J. Thorley, J. B. Asbury, D. S. Seferos, J. E. Anthony, G. D. Scholes, J. Phys. Chem. Lett. 2016, 7, 2370.

[25] T. Zeng, P. Goel, J. Phys. Chem. Lett. 2016, 7, 1351.

[26] J. Zirzlmeier, R. Casillas, S. R. Reddy, P. B. Coto, D. Lehnherr, E. T. Chernick, I. Papadopoulos, M. Thoss, R. R. Tykwinski, D. M. Guldi, Nanoscale 2016, 8, 10113.

[27] K. Miyata, Y. Kurashige, K. Watanabe, T. Sugimoto, S. Takahashi, S. Tanaka, J. Takeya, T. Yanai, Y. Matsumoto, Nat. Chem. 2017, 9, 983. 
[28] K. Bhattacharyya, A. Datta, J. Phys. Chem. C 2017, 121, 1412.

[29] S. H. Etschel, A. R. Waterloo, J. T. Margraf, A. Y. Amin, F. Hampel, C. M. Jäger, T. Clark, M. Halik, R. R. Tykwinski, Chem. Commun. 2013, 49, 6725.

[30] E. Garoni, J. Zirzlmeier, B. S. Basel, C. Hetzer, K. Kamada, D. M. Guldi, R. R. Tykwinski, J. Am. Chem. Soc. 2017, 139, 14017.

[31] D. Lehnherr, A. H. Murray, R. McDonald, R. R. Tykwinski, Angew. Chem., Int. Ed. 2010, 49, 6190.

[32] J. L. Marshall, D. Lehnherr, B. D. Lindner, R. R. Tykwinski, ChemPlusChem 2017, 82, 967.

[33] M. J. Y. Tayebjee, S. N. Sanders, E. Kumarasamy, L. M. Campos, M. Y. Sfeir, D. R. McCamey, Nat. Phys. 2017, 13, 182.

[34] P. Coppo, S. G. Yeates, Adv. Mater. 2005, 17, 3001.

[35] G. P. Miller, J. Briggs, J. Mack, Pamela A. Lord, A. Marilyn M. Olmstead, A. L. Balch, Org. Lett. 2003, 5, 4199.

[36] F. Cataldo, D. A. García-Hernández, A. Manchado, Fullerenes, Nanotubes, Carbon Nanostructures 2015, 23, 818.

[37] S. Hirata, K. Totani, J. Zhang, T. Yamashita, H. Kaji, S. R. Marder, T. Watanabe, C. Adachi, Adv. Funct. Mater. 2013, 23, 3386.

[38] J. J. Snellenburg, S. P. Laptenok, R. Seger, K. M. Mullen, I. H. M. van Stokkum, J. Stat. Softw. 2012, 49, 1.

[39] B. S. Basel, J. Zirzlmeier, C. Hetzer, B. T. Phelan, M. D. Krzyaniak, S. R. Reddy, P. B. Coto, N. E. Horwitz, R. M. Young, F. J. White, F. Hampel, T. Clark, M. Thoss, R. R. Tykwinski, M. R. Wasielewski, D. M. Guldi, Nat. Commun. 2017, 8, 15171.

[40] J. E. Anthony, J. S. Brooks, D. L. Eaton, S. R. Parkin, J. Am. Chem. Soc. 2001, 123, 9482.

[41] A. R. Waterloo, A.-C. Sale, D. Lehnherr, F. Hampel, R. R. Tykwinski, Beilstein J. Org. Chem. 2014, 10, 1692.

[42] M. Garcia-Borràs, A. Konishi, A. Waterloo, Y. Liang, Y. Cao, C. Hetzer, D. Lehnherr, F. Hampel, K. N. Houk, R. R. Tykwinski, Chem. - Eur. J. 2017, 23, 6111.

[43] J. E. Anthony, Chem. Rev. 2006, 106, 5028.

[44] M. Solà, Front. Chem. 2013, 1, 4.

[45] M. Estévez-Fregoso, J. Hernández-Trujillo, Phys. Chem. Chem. Phys. 2016, 18, 11792.

[46] A. A. Granovsky, J. Chem. Phys. 2011, 134, 214113.

[47] T. H. Dunning, P. J. Hay, in Methods on Electronic Structure Theory, Springer US, Boston, MA, 1977, pp. 1-27.

[48] J. Zirzlmeier, R. Casillas, S. R. Reddy, P. B. Coto, D. Lehnherr, E. T. Chernick, I. Papadopoulos, M. Thoss, R. R. Tykwinski, D. M. Guldi, Nanoscale 2016, 8, DOI 10.1039/c6nr02493a.

[49] S. R. Reddy, P. B. Coto, M. Thoss, J. Phys. Chem. Lett. 2018, 9, 5979.

[50] H. Nakamura, D. G. Truhlar, J. Chem. Phys. 2001, 115, 10353.

[51] H. Nakamura, D. G. Truhlar, J. Chem. Phys. 2002, 117, 5576.

[52] T. Zeng, R. Hoffmann, N. Ananth, J. Am. Chem. Soc. 2014, 136, 5755. 
[53] A. Nitzan, in Chemical Dynamics in Condensed Phases : Relaxation, Transfer and Reactions in Condensed Molecular Systems, Oxford University Press, 2006.

[54] T. C. Berkelbach, M. S. Hybertsen, D. R. Reichman, J. Chem. Phys. 2013, 138, 114103.

[55] W.-L. Chan, T. C. Berkelbach, M. R. Provorse, N. R. Monahan, J. R. Tritsch, M. S. Hybertsen, D. R. Reichman, J. Gao, X.-Y. Zhu, Acc. Chem. Res. 2013, 46, 1321.

[56] A. Kunzmann, M. Gruber, R. Casillas, R. R. Tykwinski, R. D. Costa, D. M. Guldi, Nanoscale 2018, 10, 8515.

[57] A. Kunzmann, M. Gruber, R. Casillas, J. Zirzlmeier, M. Stanzel, W. Peukert, R. R. Tykwinski, D. M. Guldi, Angew. Chem., Int. Ed. 2018, 57, 10742.

[58] T. Zeng, J. Phys. Chem. Lett. 2016, 7, 4405. 